\title{
INFLUENCIA DE LA CARGA ORGÁNICA SOBRE LA EFICIENCIA DE REACTORES RBC DE TRES ETAPAS EN EL TRATAMIENTO DE UN EFLUENTE INDUSTRIAL SINTÉTICO
}

\section{AN ORGANIC LOAD INFLUENCE UPON 3-STAGE RBC REACTORS PERFORMANCE TO TREAT A SYNTHETIC EFFLUENT}

\section{Elisabeth Behling de Calmón}

Ing. Civil., M.Sc., Dra., Profesora titular, Facultad de Ingeniería, Departamento de Ingeniería Sanitaria y Ambiental (DISA), Universidad del Zulia, Maracaibo, Venezuela elisabeth.behling@gmail.com

Julio César Marín Leal

Biólogo, M.Sc., Dr., Profesor titular, Facultad de Ingeniería, Departamento de Ingeniería Sanitaria y Ambiental (DISA), Universidad del Zulia, Maracaibo, Venezuela jmarin@fing.luz.edu.ve

Ana Chirinos

Ing. Civil., Facultad de Ingeniería, Departamento de Ingeniería Sanitaria y Ambiental (DISA), Universidad del Zulia, Maracaibo, Venezuela

Nancy Rincón Lizardo

Ing. Civil., M.Sc., Dra., Profesora titular, Facultad de Ingeniería, Departamento de Ingeniería Sanitaria y Ambiental (DISA), Universidad del Zulia, Maracaibo, Venezuela

ncrincon1@gmail.com

Gilberto Colina Andrade

Lcdo., M.Sc., Dr., Profesor titular, Facultad de Ingeniería, Departamento de Ingeniería Sanitaria y Ambiental (DISA), Universidad del Zulia, Maracaibo, Venezuela

gjcolina@hotmail.com

Johan Mesa ${ }^{\dagger}$

Téc. Medio, Lcdo., Auxiliar docente, Facultad de Ingeniería, Ciclo Básico, Departamento de Química, Universidad del Zulia, Maracaibo, Venezuela

Fecha de recepción: 19 de marzo de 2012

Fecha de aprobación: 15 de diciembre de 2012 


\title{
RESUMEN
}

El presente artículo incluye el estudio de la influencia de la carga orgánica (CO) sobre la eficiencia de reactores biológicos rotativos de contacto (RBC), aerobios de tres etapas, al tratar un efluente industrial sintético, con la finalidad de establecer la adecuación del efluente final con respecto a los límites de descarga establecidos en la normativa de Venezuela. Durante la experimentación, se evaluaron $\mathrm{pH}$, alcalinidad total, oxígeno disuelto, demanda biológica de oxígeno (DBO), demanda química de oxígeno (DOO), sólidos suspendidos totales (SST), nitrógeno total Kjeldahl (NTK), $\mathrm{NH}_{4}{ }^{+}, \mathrm{NO}_{2}^{-}$y $\mathrm{NO}_{3}$; de acuerdo con los métodos estándares. La variación de la $\mathrm{CO}$ aplicada se obtuvo mediante modificación del tiempo de retención hidráulico (TRH), (24, 12 y 6 h), y se mantuvo constante la DQO de entrada (influente sintético de sacarosa + urea). La mayor eficiencia de remoción de DQO se obtuvo para un CO global de 11,68 gDOO/m².d (96,25\%; TRH=12 h). Para los TRH 24 y 12 h, la eficiencia de remoción global de $\mathrm{N}$-total fue de 66,92 y 62,95\%, respectivamente. La mayor remoción de $\mathrm{C}$ y de $\mathrm{N}$ se obtuvo en la primera etapa de los reactores y se logró cumplir con el límite venezolano permisible de descarga para DOO (<350 mg/L). La posible ocurrencia del proceso de nitrificación repercutió sobre el aumento de las concentraciones de nitrógeno inorgánico en el efluente final.

Palabras clave: aguas residuales industriales, carga orgánica, reactor RBC, tiempo de retención hidráulico, tratamiento biológico.

\begin{abstract}
This paper deals with the organic load rate (OLR) influence upon removal efficiency of aerobic rotating biological contactor (RBC), i.e. 3-stage reactors by treatment of an industrial synthetic effluent in order to establish adjustment of closing effluent in compliance with Venezuelan legislation. Upon experiment and based on standard methods we evaluated $\mathrm{pH}$, total alkalinity, dissolved oxygen, biological oxygen demand (BOD), chemical oxygen demand (COD), total suspended solid (TSS), overall Kjeldahl nitrogen (TKN), $\mathrm{NH}_{4}{ }^{+}, \mathrm{NO}_{2}{ }^{-}$and $\mathrm{NO}_{3}{ }^{-}$. A variation of OLR applied was obtained by modifying hydraulic retention time (HRT) $(24,12$, and 6 h), staying constant the initial COD (synthetic influent of sucrose + urea.) The greater efficiency of COD removal was got for an overall OLR of $11.68 \mathrm{gCOD} / \mathrm{m}^{2}$.d (96.25\%, HRT=12 h.) Removal efficiencies of overall N-total in HRT 24 and $12 \mathrm{~h}$ were 66.92 and $62.95 \%$, respectively. The major $C \& N$ removal was got during the first stage of reactors to comply with Venezuelan COD allowable limit ( $<350 \mathrm{mg} / \mathrm{L}$ ). A likely occurrence of nitrification process triggered increased inorganic nitrogen concentrations by final effluent.
\end{abstract}

Keywords: biological treatment, hydraulic retention time, industrial wastewaters, organic load rate, $\mathrm{RBC}$ reactor. 


\section{INTRODUCCIÓN}

La materia orgánica disuelta en los efluentes industriales constituye la mayor amenaza a los recursos acuáticos receptores tales como: arroyos, ríos, lagos y océanos. La descarga de estos efluentes puede conllevar a la muerte de peces y otras especies marinas, los cuales durante su descomposición demandan una cantidad apreciable de oxígeno disuelto, y disminuyen su concentración a valores intolerables [1 1, 21, 23], alterando de esta manera el uso potencial del recurso agua. Estas descargas producen además, el crecimiento acelerado de plantas acuáticas, originando el problema de eutrofización o envejecimiento prematuro del cuerpo de agua $[14,17]$. Para solucionar problemas de este tipo, se suele diseñar y construir plantas de tratamiento con el propósito de remover los contaminantes de los caudales de las aguas residuales, de tal manera, que el efluente tratado sea seguro para ser descargado al ambiente, cumpliendo con la normativa legal establecida.

Los sistemas de tratamiento biológico han mostrado ser altamente eficientes en la remoción de la carga contaminante de un variado tipo de industrias [23, 24, 34, 35]. En particular, los reactores biológicos rotativos de contacto (RBC, según sus siglas en inglés), también llamados biodiscos, son un tipo de tratamiento de crecimiento adherido, que posee un número significativo de ventajas sobre otros sistemas de tratamiento; efluentes de buena calidad incluida la nitrificación total, bajo costo, facilidad de operación y mantenimiento [13, 31, 34], resultando una tecnología muy atractiva desde el punto de vista económico y ambiental. Los reactores RBC de multietapas han sido utilizados para degradar una amplia variedad de sustancias presentes en residuos, domésticos e industriales [1, 2, 4, 5, 12, 18, 24, 28, 33, 34]. El interés de la compartimentalización está basado en el aumento de la remoción de la demanda bioquímica de oxígeno (DBO), y del nitrógeno amoniacal $\left(\mathrm{NH}_{4}^{+}\right)$[7].

En el presente estudio, se evaluó la influencia de la carga orgánica (CO) sobre la eficiencia de reactores RBC aerobios de tres etapas, con el fin de establecer la adecuación del efluente final con respecto de los límites de descarga establecidos en la normativa de Venezuela.

\section{MATERIALES Y MÉTODOS}

A continuación, se describen los materiales y métodos empleados en la presente investigación, para evaluar la influencia de la CO sobre la eficiencia de reactores RBC aerobios de tres etapas, bajo condiciones de laboratorio.

\subsection{EQUIPO EXPERIMENTAL}

Se emplearon dos réplicas de reactores biológicos tipo biodiscos de tres etapas (cámaras), en condiciones mesofílicas, en los cuales el flujo se desplazaba de manera perpendicular a los discos (Figura 1). 


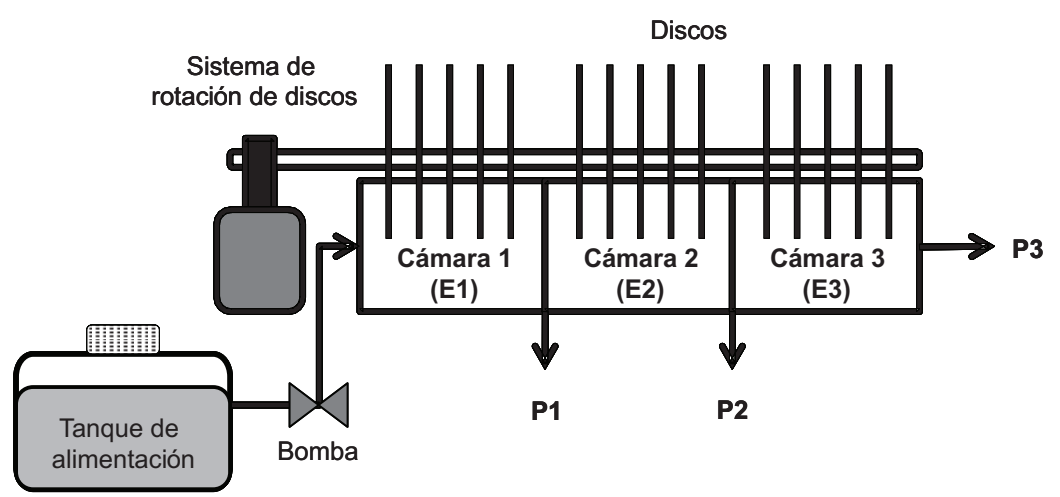

Figura 1. Esquema del sistema de tratamiento aerobio conformado por reactores biológicos rotativos de contacto (RBC), de tres etapas. P1, P2 y P3: puntos de muestreo de efluente tratado. E1, E2 y E3: efluentes de las cámaras 1, 2 y 3 , respectivamente

Cada cámara estaba delimitada por una pantalla perforada que permite el flujo de efluente entre las cámaras. El grado de sumergencia de los discos estaba cerca del $40 \%$. Tanto el tanque como los discos se fabricaron en acrílico transparente. El eje, las tuercas y arandelas fueron de acero inoxidable para evitar la corrosión. Las características generales de los reactores se presentan en la Tabla 1.

Tabla 1. Dimensiones y características de los reactores biológicos rotativos de contacto (RBC) aerobio de tres etapas, empleados en la investigación

\begin{tabular}{|l|l|}
\hline \multicolumn{1}{|c|}{ Característica } & \multicolumn{1}{|c|}{ Valor } \\
\hline Diámetro del disco $(\mathrm{cm})$ & 18 \\
\hline Diámetro del tanque $(\mathrm{cm})$ & 23 \\
\hline Área total de contacto $\left(\mathrm{m}^{2}\right)$ & 1,836 \\
\hline Porcentaje de sumergencia $(\%)$ & 40 \\
\hline Número de etapas & 3 \\
\hline Número de discos por etapa & 12 \\
\hline Espaciamiento entre discos $(\mathrm{m})$ & 0,01 \\
\hline Diámetro del eje (pulg) & $3 / 8^{\prime \prime}$ \\
\hline Longitud total del reactor $(\mathrm{cm})$ & 61 \\
\hline Volumen útil del reactor (L) & 9 \\
\hline Velocidad de giro del disco (rpm) & 10 \\
\hline Tiempo de retención hidráulico (h) & $24,12,6$ \\
\hline
\end{tabular}

La rotación de los discos fue garantizada mediante el uso de un motor eléctrico marca Cole-Parmer, acoplado a una serie de engranajes y poleas que ayudaban al reductor de voltaje a controlar y regular la velocidad de rotación requerida $(10$ rpm). El afluente era bombeado al reactor mediante una bomba peristáltica marca Cole-Parmer. Cada sistema contaba con tres puntos de muestreo del efluente en tratamiento, ubicados a la salida de cada etapa o cámara (incluida la salida final: P1, P2 y P3; Figura 1). 


\subsection{EFLUENTE}

Para esta investigación, se preparó un efluente industrial sintético que contenía sacarosa (azúcar común), como fuente de carbono y úrea comercial como fuente de nitrógeno, a una proporción $\mathrm{C}: \mathrm{N} \approx 5$, para garantizar la disponibilidad de $\mathrm{C}$ hasta la última cámara de los reactores [14]. La composición detallada del efluente sintético se presenta en la Tabla 2, la cual se ajusta de cierta manera a la de efluentes de industrias alimenticias $[26,30]$.

Tabla 2. Composición del efluente industrial sintético usado en los sistemas RBC aerobios de tres etapas

\begin{tabular}{|c|l|}
\hline Compuesto & $\mathbf{~ m g / L}$ \\
\hline sacarosa & 1.000 \\
\hline Úrea & 400 \\
\hline $\mathrm{NaHCO}_{3}$ & 1.500 \\
\hline $\mathrm{Na}_{2} \mathrm{HPO}_{4}$ & 75 \\
\hline $\mathrm{KH}_{2} \mathrm{PO}_{4}$ & 37,5 \\
\hline $\mathrm{MgSO}_{4}$ & 25 \\
\hline
\end{tabular}

Fuente: modificado de Gupta y Gupta, 1999

\subsection{CONTROL Y ANÁLISIS DEL SISTEMA}

El inóculo de microorganismos (lodo), para los RBC aerobios de tres etapas, se obtuvo de una planta de tratamiento aerobio para aguas residuales domésticas de la región zuliana (Venezuela). Este lodo fue mezclado con el efluente industrial sintético y dispuesto dentro del reactor para estimular el crecimiento de la microflora sobre los discos. Los sistemas fueron alimentados de manera discontinua a un tiempo de retención hidráulico (TRH), de 24 h con efluente sintético, hasta observar una carga microbiana adecuada y lograr la estabilidad de los parámetros fisicoquímicos. Posteriormente, Ios RBC operaron bajo la modalidad de flujo continuo, y se establecieron las condiciones que se describen en la Tabla 3. Cada etapa experimental se extendió hasta alcanzar las condiciones de equilibrio en los parámetros fisicoquímicos monitoreados en el efluente final. El efecto de la CO aplicada sobre el desempeño de los RBC de tres cámaras, fue evaluado por la disminución del TRH $(24,12$ y 6 h), y se mantuvo constante la DOO del influente.

Tabla 3. Fases de experimentación en los sistemas RBC aerobios de tres etapas, alimentados con el efluente industrial sintético (sacarosa + úrea). CO: carga orgánica

\begin{tabular}{|c|c|c|c|c|c|}
\hline Etapa & $\begin{array}{c}\text { Días de } \\
\text { experimentación }\end{array}$ & $\begin{array}{c}\mathrm{CO}_{\text {aplicada en }} 1^{\text {ra }} \text { cámara } \\
\left(g D Q O_{\top} / m^{2} . d\right)\end{array}$ & $\begin{array}{l}\mathrm{CO}_{\text {aplicada }} \text { global } \\
\left(\mathrm{gDOO} / \mathrm{m}^{2} . \mathrm{d}\right)\end{array}$ & $\begin{array}{c}\text { TRH } \\
\text { (h) }\end{array}$ & $\begin{array}{l}\text { Rotación de } \\
\text { discos (rpm) }\end{array}$ \\
\hline 1 & 31 & 18,31 & 6,10 & 24 & 10 \\
\hline II & 18 & 35,03 & 11,68 & 12 & 10 \\
\hline III & 29 & 71,90 & 23,97 & 6 & 10 \\
\hline
\end{tabular}


Diariamente se efectuaron mediciones de oxígeno disuelto (OD), temperatura, pH y alcalinidad total en las entradas (influente), y en las salidas (efluentes), de las cámaras de cada RBC (P1, P2 y P3; Figura 1). Tres veces por semana, se hicieron mediciones de $\mathrm{NO}_{2}^{-}, \mathrm{NO}_{3}{ }^{-}, \mathrm{NH}_{4}{ }^{+}$, nitrógeno total Kjeldahl (NTK), demanda química de oxígeno total $\left(\mathrm{DOO}_{\mathrm{T}}\right)$ y soluble ( $\left.\mathrm{DOO}_{\mathrm{S}}\right), \mathrm{DBO}_{5,20}$ y sólidos suspendidos totales (SST). Todos los métodos de análisis empleados se siguieron de acuerdo con APHA et al [3].

\section{RESULTADOS Y DISCUSIÓN}

Los resultados generales del presente estudio, empleando los sistemas RBC aerobios de tres etapas, se presentan en la Tabla 4 . La temperatura media de experimentación fue de $25,7 \pm 1,6^{\circ} \mathrm{C}$.

\section{1. pH Y ALCALINIDAD TOTAL}

Los valores de $\mathrm{pH}$ y alcalinidad total variaron ligeramente en cada etapa experimental (Tabla 4). Se puede apreciar que no se presentaron diferencias significativas del $\mathrm{pH}$ en las cámaras $(p<0,001)$. El incremento de la CO no afectó de manera significativa su comportamiento en el sistema de tratamiento que se mantuvo en un valor medio de 8,4 en el efluente final. Los valores de $\mathrm{pH}$ permanecieron dentro del rango recomendado en la literatura para este tipo de tratamiento [20], lo cual garantiza la supervivencia de una comunidad microbiana mixta sobre los discos. Estos valores también cumplieron con los límites de descarga establecidos por la legislación de Venezuela (entre 6 y 9) [8].

Por su parte, la alcalinidad total, fue suficiente para amortiguar la posible disminución del pH debida a la oxidación de la materia orgánica y del amoníaco [31]. Se observó una relación inversa entre la alcalinidad total y la concentración de $\mathrm{NO}_{3}^{-}$en el sistema durante todas las etapas experimentales. Adicionalmente, cuando la alcalinidad total disminuía desde la cámara 1 hasta la última cámara, se observó un aumento en la concentración de $\mathrm{NO}_{3}$ - en este mismo sentido. Este comportamiento concuerda con Nowak [25], que reportó que una baja alcalinidad en los efluentes de reactores RBC puede ser producida por la falta de desnitrificación. Gupta y Gupta [14], observaron el mismo comportamiento y lo atribuyeron a un fenómeno de nitrificación y desnitrificación simultánea, lo cual será discutido posteriormente en este mismo trabajo.

\subsection{OXÍGENO DISUELTO Y DEMANDA BIOQUÍMICA DE OXÍGENO}

De manera general las concentraciones de OD se mantuvieron mas o menos constantes dentro de las cámaras, y disminuyeron notablemente en la primera cámara del reactor, en especial el TRH 6 h (Tabla 4). Estos valores fueron muy diferentes entres las cámaras y para todas las cargas orgánicas aplicadas. La reducción del contendido de OD es producto de la oxidación de la materia orgánica presente en el influente. Usualmente, como consecuencia de una respiración activa en las primeras etapas de los RBC, la concentración del OD alcanza niveles mínimos, y se incrementa 
a lo largo del reactor donde la concentración del sustrato es menor [7]. Cuando el reactor operó a TRH 24 h, se consumió una parte del OD en la primera cámara (mostrado por la remoción de $\mathrm{DBO}_{5,20}$ ), pero hubo tiempo de reoxigenación (de 6,01 a 6,12 mg/L), debido a un mayor tiempo de contacto [7]. Estudios con sistemas RBC han revelado que un mayor tiempo de contacto mejora la difusión del sustrato en el biofilm y su consiguiente remoción del influente [16, 22, 24]. Najafpour et al [24], y Chen et al, [6], reportaron un comportamiento similar del OD en reactores RBC de tres etapas, durante el tratamiento de efluentes de comida enlatada y de aguas residuales municipales, respectivamente.

Tabla 4. Valores medios y desviaciones estándares de los parámetros fisicoquímicos evaluados durante el tratamiento del efluente industrial sintético (sacarosa + úrea), en los sistemas RBC aerobios de tres etapas, con respecto del tiempo de retención hidráulico aplicado (TRH), y las cámaras (E1, E2 y E3). I: influente, E1: efluente de la cámara 1, E2: efluente de la cámara 2, E3: efluente de la cámara 3 (efluente final), AT: alcalinidad total, OD: oxígeno disuelto, $\mathrm{n}=31$ (TRH=24 h), $\mathrm{n}=18$ (TRH=12 h), $\mathrm{n}=29$ (TRH=6 h)

\begin{tabular}{|c|c|c|c|c|c|c|c|c|c|c|c|c|}
\hline Etapa & $\begin{array}{c}\text { TRH } \\
\text { (h) }\end{array}$ & $\mathrm{pH}$ & $\begin{array}{c}\mathrm{AT} \\
\left(\mathrm{mgCaCO}_{3} / \mathrm{L}\right)\end{array}$ & $\begin{array}{c}\text { OD } \\
\text { (mg/L) }\end{array}$ & $\begin{array}{l}\mathrm{DBO}_{5,20} \\
\text { (mg/L) }\end{array}$ & $\begin{array}{l}\mathrm{DOO}_{\mathrm{T}} \\
(\mathrm{mg} / \mathrm{L})\end{array}$ & $\begin{array}{l}\mathrm{DOO}_{\mathrm{s}} \\
\text { (mg/L) }\end{array}$ & $\begin{array}{c}\text { SST } \\
\text { (mg/L) }\end{array}$ & $\begin{array}{l}\mathrm{NO}_{2}^{-} \\
\text {(mg/L) }\end{array}$ & $\begin{array}{c}\mathrm{NO}_{3}^{-} \\
\text {(mg/L) }\end{array}$ & $\begin{array}{l}\mathrm{NH}_{4}^{+} \\
(\mathrm{mg} / \mathrm{L})\end{array}$ & $\begin{array}{l}\text { N-total } \\
\text { (mg/L) }\end{array}$ \\
\hline I & 24 & $8,02 \pm 0,45$ & $975,4 \pm 43,5$ & $6,01 \pm 0,84$ & $999,39 \pm 9,11$ & $1.244,86 \pm 93,43$ & $1.126,44 \pm 3,54$ & $12,8 \pm 1,6$ & $1,34 \pm 0,12$ & $1,89 \pm 0,25$ & $17,53 \pm 11,26$ & $215,90 \pm 6,45$ \\
\hline $\mathrm{E} 1$ & & $8,26 \pm 0,34$ & $960,9 \pm 87,5$ & $6,12 \pm 0,67$ & $21,27 \pm 8,39$ & $81,32 \pm 4,12$ & $76,45 \pm 19,48$ & $68,6 \pm 8,2$ & $26,54 \pm 8,47$ & $22,79 \pm 10,37$ & $5,87 \pm 0,72$ & $55,83 \pm 12,32$ \\
\hline E2 & & $8,40 \pm 0,33$ & $930,2 \pm 102,6$ & $6,82 \pm 0,44$ & $23,15 \pm 12,23$ & $66,57 \pm 11,45$ & $58,86 \pm 22,32$ & $92,4 \pm 5,4$ & $29,29 \pm 6,85$ & $31,07 \pm 8,62$ & $4,20 \pm 0,86$ & $65,18 \pm 10,69$ \\
\hline E3 & & $8,44 \pm 0,34$ & $920,9 \pm 106,5$ & $6,78 \pm 0,41$ & $26,81 \pm 14,49$ & $90,22 \pm 4,25$ & $76,47 \pm 28,35$ & $118,1 \pm 8,5$ & $30,42 \pm 6,87$ & $36,46 \pm 8,58$ & $3,94 \pm 0,88$ & $71,43 \pm 10,46$ \\
\hline I & 12 & $8,12 \pm 0,26$ & $972,9 \pm 109,2$ & $6,34 \pm 1,09$ & $985,75 \pm 12,65$ & $1.190,93 \pm 85,97$ & $1.096,14 \pm 4,57$ & $14,2 \pm 1,3$ & $1,22 \pm 0,20$ & $2,01 \pm 0,33$ & $17,24 \pm 8,24$ & $226,60 \pm 4,21$ \\
\hline E1 & & $8,19 \pm 0,15$ & $995,5 \pm 64,9$ & $3,74 \pm 1,11$ & $30,63 \pm 2,70$ & $48,94 \pm 9,35$ & $44,09 \pm 15,84$ & $224,7 \pm 8,0$ & $3,91 \pm 1,30$ & $36,35 \pm 2,96$ & $7,11 \pm 1,27$ & $80,70 \pm 8,30$ \\
\hline E2 & & $8,34 \pm 0,16$ & $937,7 \pm 80,8$ & $4,40 \pm 0,47$ & $37,24 \pm 5,69$ & $42,73 \pm 14,90$ & $29,31 \pm 16,79$ & $214,2 \pm 7,1$ & $7,07 \pm 1,08$ & $42,11 \pm 8,30$ & $5,66 \pm 1,10$ & $80,49 \pm 8,72$ \\
\hline E3 & & $8,34 \pm 0,10$ & $924,6 \pm 59,8$ & $4,42 \pm 0,26$ & $43,53 \pm 1,44$ & $44,92 \pm 6,83$ & $41,19 \pm 28,16$ & $206,0 \pm 9,5$ & $15,67 \pm 2,30$ & $45,20 \pm 7,33$ & $4,98 \pm 1,40$ & $83,96 \pm 7,79$ \\
\hline I & 6 & $8,18 \pm 0,06$ & $970,0 \pm 54,3$ & $6,22 \pm 1,20$ & $1.002,32 \pm 6,21$ & $1.222,23 \pm 87,81$ & $1.130,01 \pm 2,66$ & $15,1 \pm 1,8$ & $1,28 \pm 0,25$ & $1,93 \pm 0,66$ & $17,68 \pm 7,32$ & $221,74 \pm 7,33$ \\
\hline $\mathrm{E} 1$ & & $8,28 \pm 0,09$ & $1.190,5 \pm 77,8$ & $0,09 \pm 0,03$ & $56,32 \pm 3,41$ & $153,57 \pm 22,67$ & $102,21 \pm 45,03$ & $245,2 \pm 10,6$ & $1,51 \pm 0,41$ & $1,99 \pm 0,64$ & $73,01 \pm 5,10$ & $87,31 \pm 5,19$ \\
\hline E2 & & $8,37 \pm 0,06$ & $1.160,0 \pm 69,1$ & $1,35 \pm 0,72$ & $63,22 \pm 4,11$ & $75,47 \pm 16,60$ & $68,77 \pm 15,65$ & $230,6 \pm 12,8$ & $3,35 \pm 1,02$ & $52,92 \pm 13,24$ & $63,04 \pm 4,30$ & $136,98 \pm 14,22$ \\
\hline E3 & & $8,38 \pm 0,06$ & $1.112,5 \pm 44,4$ & $3,38 \pm 0,50$ & $61,34 \pm 6,88$ & $66,87 \pm 13,61$ & $57,33 \pm 14,43$ & $222,1 \pm 23,3$ & $8,96 \pm 3,44$ & $261,27 \pm 112,88$ & $54,26 \pm 6,67$ & $340,55 \pm 111,28$ \\
\hline
\end{tabular}

El OD es determinante en la oxidación del carbono y la nitrificación, y es el factor de diseño más importante en reactores RBC aerobios. Para prevenir que sea un factor limitante, la concentración de OD debe ser de al menos $2 \mathrm{mg} / \mathrm{L}$ en las primeras etapas de estos sistemas [25]. En tal sentido y considerando los resultados del presente estudio, la remoción de materia orgánica, así como la dinámica de las formas de $\mathrm{N}$ pudieron verse perturbadas (baja remoción), por la poca disponibilidad de OD, específicamente para el TRH 6 h, cuyos valores se ubicaron en 0,09 $\pm 0,03 ; 1,35 \pm 0,72$ y 3,38 $\pm 0,50$ mg/L, para las cámaras 1, 2 y 3, respectivamente (Tabla 4).

En cuanto a la $\mathrm{DBO}_{5,20^{\prime}}$ se observó la más alta remoción en la primera cámara para el TRH 24 h (97,9\% de eficiencia), producto de la actuación de bacterias heterótrofas [20], y se obtuvo concentraciones por debajo del límite exigido por la normativa venezolana para este parámetro 160 mg/L) [8]. Sin embargo, debido al aporte de la biomasa desprendida de los biodiscos, la $\mathrm{DBO}_{5,20}$ se incrementó ligeramente en las cámaras subsiguientes (Tabla 4). La baja concentración de materia orgánica biodegradable en las cámaras 2 y 3, favoreció el proceso de nitrificación. 
Para la mayor CO aplicada (TRH 6 h), los efluentes de las cámaras 2 y 3 (E2 y E3), mostraron una $\mathrm{DBO}_{5,20}$ ligeramente superior a la establecida en la legislación para descarga en cuerpos de agua naturales $(63,22 \pm 4,11$ y $61,34 \pm 6,88 \mathrm{mg} / \mathrm{L}$, respectivamente). Sin embargo, durante el tratamiento con sólo la primera cámara se cumplía con esta exigencia. Najafpour et al [24], en su investigación con un sistema RBC de tres etapas, concluyeron que un reactor de etapa simple puede ser suficiente para aplicaciones prácticas.

\subsection{DEMANDA QUÍMICA DE OXÍGENO}

La Figura 2 muestra la variación de la $\mathrm{DOO}_{\mathrm{T}}$ a la salida de cada cámara del reactor (E1, E2 y E3), durante el tratamiento, con respecto del TRH aplicado.
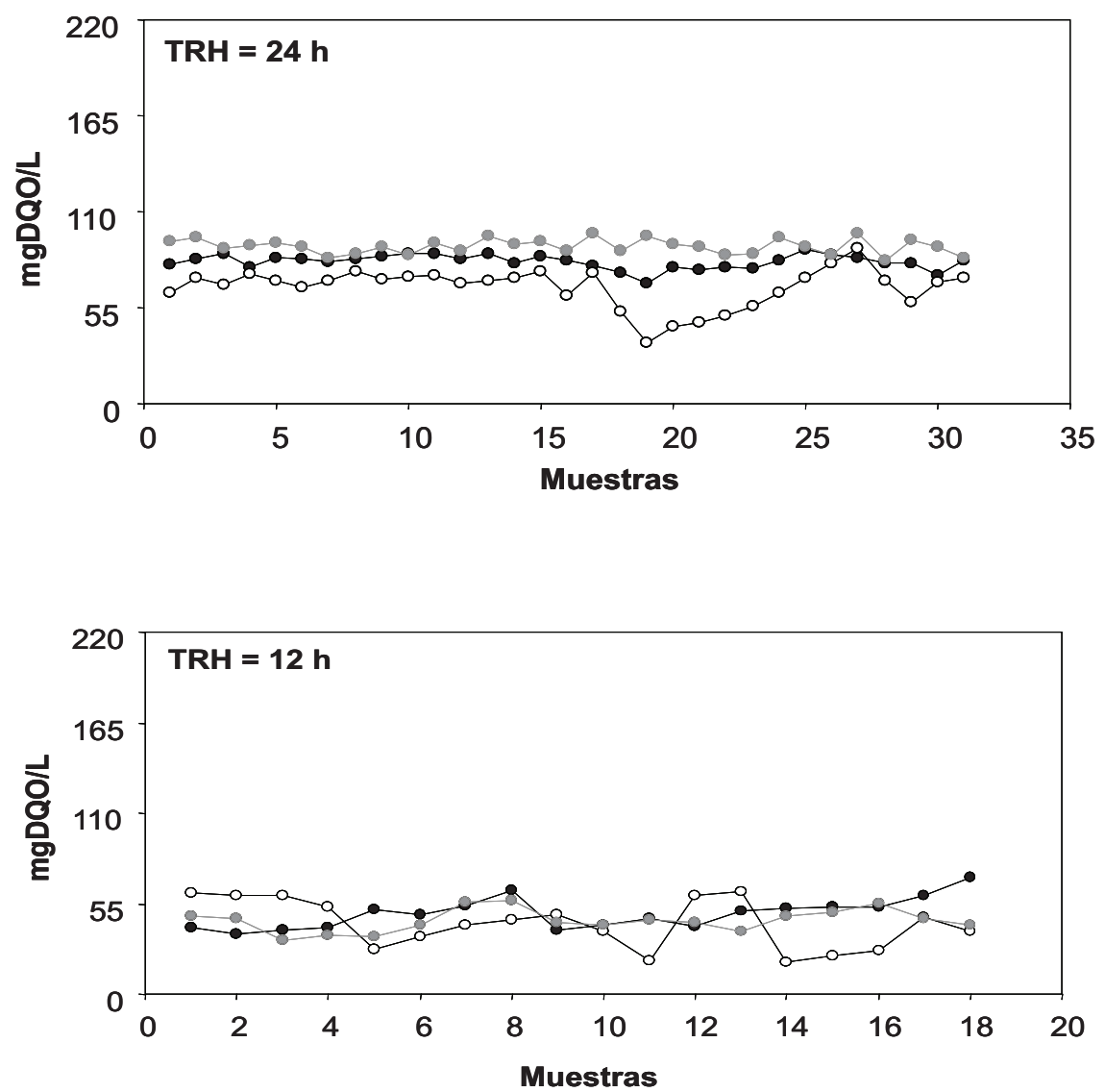


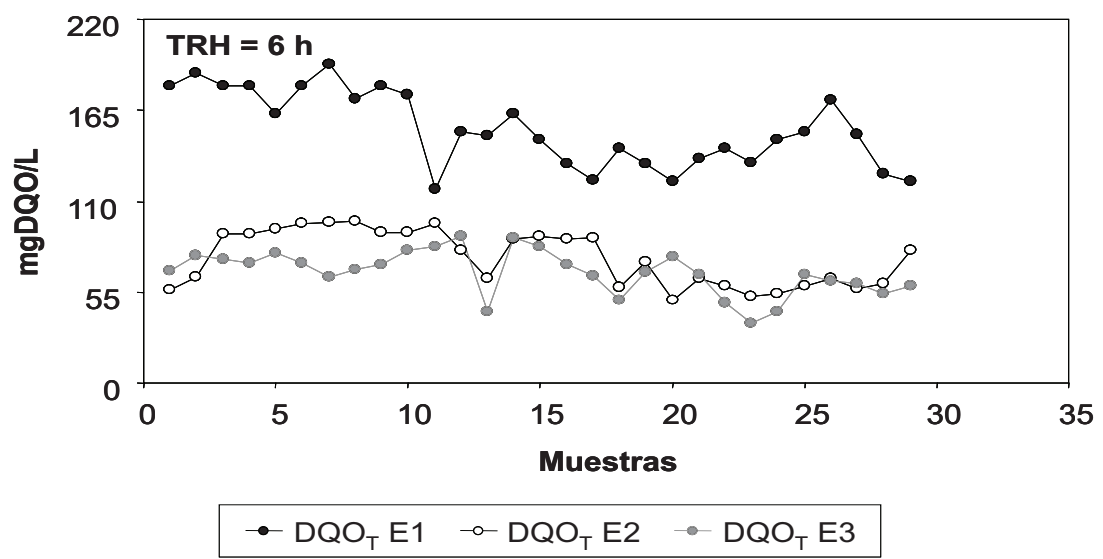

Figura 2. Variación de la demanda química de oxígeno total $\left(D_{0} \mathrm{O}_{\mathrm{T}}\right)$ a la salida de cada cámara (E1, E2 y E3), durante el tratamiento del efluente sintético (sacarosa + úrea) en los sistemas RBC aerobios de tres etapas, con respecto al tiempo de retención hidráulico aplicado (TRH)

El comportamiento de la $\mathrm{DOO}_{\mathrm{T}}$ fue similar al de la $\mathrm{DBO}_{5,20}$. Se obtuvo una elevada remoción de la materia orgánica en la primera cámara, para todos los TRH aplicados (Tabla 4 y Figura 2). Cuando el sistema operó a 24 y 12 h de TRH (CO aplicada de 6, 10 y 11,68 gDOO $/ \mathrm{m}^{2}$.d, respectivamente, Tabla 3), se removió prácticamente todo el sustrato en la primera cámara. Las contribuciones de la segunda cámara a la eficiencia de remoción de DQO, fueron cercanas al 1\%, debido al bajo contenido de $\mathrm{C}$ en el sustrato.

Cuando el sistema operó a la mayor CO ensayada (TRH 6 h), se removieron 1.060 mgDOO $/ \mathrm{L}$ en la primera cámara, y originaron un efluente (E1), con una concentración de 153,57 mgDQO $/ \mathrm{L}$ (remoción 87,44\%), y la eficiencia de remoción de cámara $1+2$ fue de 93,83\%. El incremento de la $\mathrm{DOO}_{\mathrm{T}}$ en el efluente de la cámara 3, resultó del aumento de la cantidad de sólidos en E3 (Tabla 4), producto del desprendimiento de la biopelícula de los discos. A medida que aumentaba la $\mathrm{CO}$ en el reactor, se observó un incremento en el espesor de la biopelícula que alcanzó su máximo grosor, cuando el sistema operó al menor TRH, comportamiento éste muy difundido en la literatura revisada $[7,21,27,32]$.

La mayor eficiencia de remoción de $\mathrm{DQO}_{\mathrm{T}}$ se logró en el TRH 12 h, obteniéndose una eficiencia global de 96,25\%. La primera cámara representó un 95,89\% de remoción de la $\mathrm{DOO}_{\mathrm{T}}$ en el sistema de tratamiento.

Con respecto de la $\mathrm{DQO}_{\boldsymbol{s}^{\prime}}$ las $\mathrm{CO}$ aplicadas en la primera cámara fueron 16,57; 32,24 y 66,47 gDOO $/ \mathrm{m}^{2} . d$, mientras que las CO globales se ubicaron en 5,52; 10,75 y 22, $16 \mathrm{gDOO} / \mathrm{m}^{2} . d$ para los TRH 24, 12 y 6 h, respectivamente. Este parámetro tuvo un comportamiento similar a 
la $\mathrm{DQO}_{\top}$, alcanzando altas eficiencias de remoción en la primera cámara $(93,21 ; 95,98$ y 90,95\% para TRH de 24, 12 y $6 \mathrm{~h}$, respectivamente).

El efluente generado en la primera cámara del reactor (E1), ya cumplía con las exigencias de la normativa legal venezolana para la descarga en cuerpos de agua naturales, mostrando una concentración de DOO muy por debajo de 350 mg/L [8]. Por tal motivo y similar al comportamiento de la $\mathrm{DBO}_{5,20^{\prime}}$ un reactor de una sola cámara sería suficiente para tratar un efluente real con características similares al utilizado en el presente estudio.

Di Palma y Verdone [9], reportaron que el incremento de la CO aplicada, disminuye la eficiencia de remoción de sustratos carbonosos. Este comportamiento ha sido reportado en otros estudios en los cuales se observa por lo general, una disminución del porcentaje de remoción del sustrato cuando se incrementa la CO y/o la carga hidráulica [10, 18, 19]. Najafpour et al [24], estudiando un reactor de tres cámaras, observaron que a medida en que se incrementaba la CO, decrecía la eficiencia de remoción de DOO. Estos investigadores concluyeron que la reducción de la eficiencia de remoción muestra insuficiente capacidad de eliminación, debido a condiciones limitantes en la transferencia de oxígeno en el biofilm. También observaron que el primer compartimiento fue el más eficiente en la remoción de DQO, obteniendo eficiencias de 73,4 y 88,8\% para TRH de 24 y $48 \mathrm{~h}$, respectivamente. La CO aplicada en la primera etapa fue de 55,33 y $110,66 \mathrm{gDOO} / \mathrm{m}^{2} \cdot \mathrm{d}$, para los TRH antes indicados.

Al comparar los resultados obtenidos entre la presente experimentación y lo reportado por Najafpour et al [24], se puede decir que la eficiencia de remoción se incrementó para las dos primeras cargas aplicadas. Sin embargo, disminuyó cuando el TRH fue de $6 \mathrm{~h}$. Esto se evidenció también por la disminución brusca del OD en la primera cámara, al alcanzar valores de 0,09 mg/L, por lo cual para la mayor CO aplicada, se puede inferir que hubo limitaciones en la transferencia de oxígeno y por esta razón, disminuyó la eficiencia de remoción.

\subsection{NITRÓGENO}

La Tabla 4 muestra los valores medios de las concentraciones $\mathrm{NO}_{2}{ }_{2}, \mathrm{NO}_{3}{ }^{-}, \mathrm{NH}_{4}{ }^{+}$y $\mathrm{N}$-total durante la etapa experimental. En las Figuras 3, 4 y 5, se presentan las variaciones a la salida de cada cámara (E1, E2 y E3), con respecto del TRH aplicado. El contenido de nitrógeno en el influente de los RBC, correspondió en su mayoría a nitrógeno orgánico, el cual fue hidrolizado rápidamente por los microorganismos presentes en la biopelícula para transformarlo a $\mathrm{NO}_{2}{ }_{2}^{-}, \mathrm{NO}_{3}^{-} \mathrm{y} / \mathrm{O} \mathrm{NH}_{4}{ }^{+}$[29].

En la presente investigación, la mayor parte del nitrógeno total $(215,90 \mathrm{mg} / \mathrm{L})$ del influente, correspondía a nitrógeno orgánico (195,14 mg/L). Los resultados del tratamiento muestran una gran disminución de las formas orgánicas en la primera etapa, alcanzando una eficiencia de remoción de 99,68\% para el TRH 24 h (carga aplicada en la primera cámara 2,87 gN-org/m².d). La concentración de nitrógeno orgánico en E1 fue de 0,63 mg/L. 

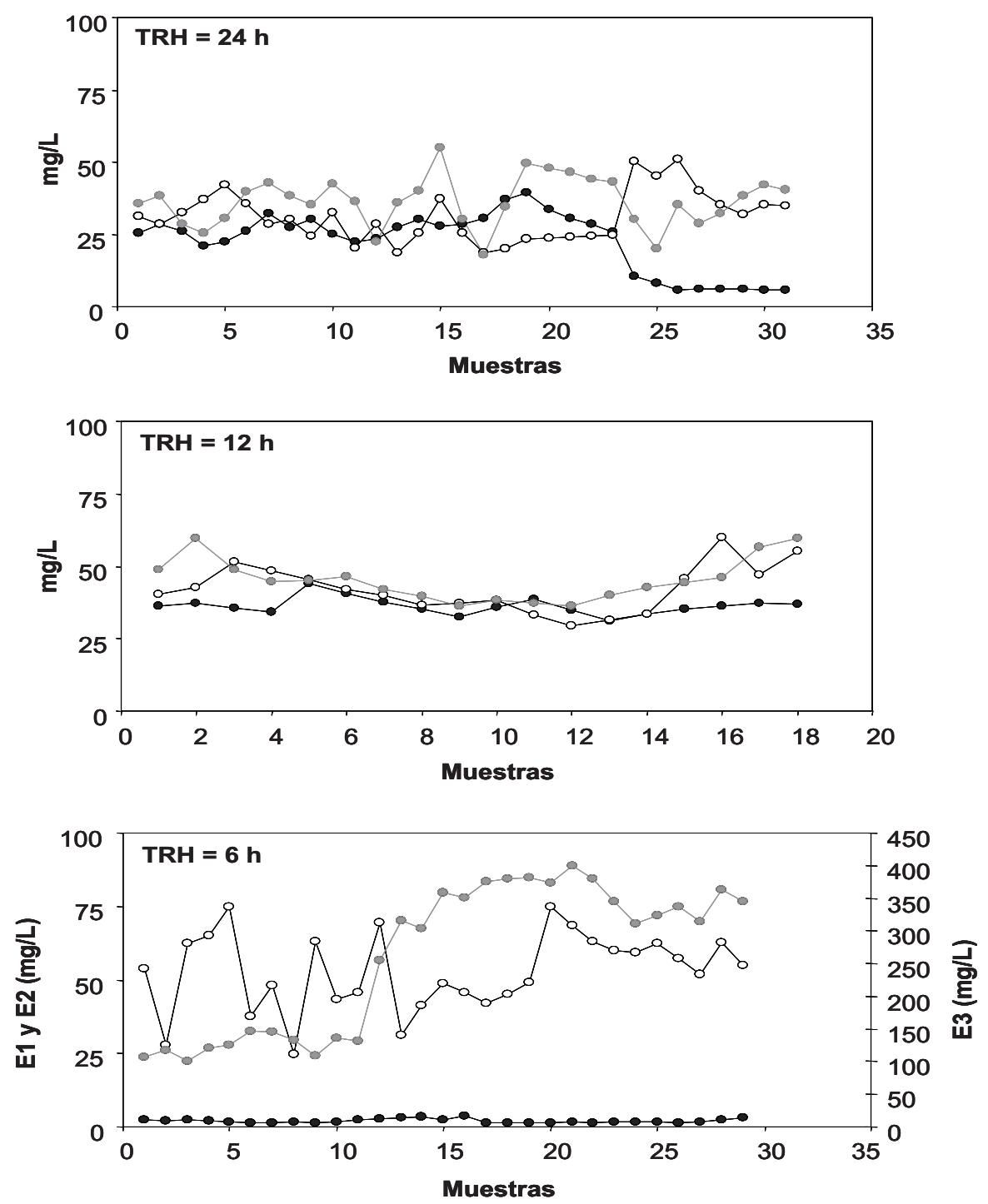

$-\mathrm{NO}_{3}{ }^{-} \mathrm{E} 1-\mathrm{NO}_{3}^{-} \mathrm{E} 2-\mathrm{NO}_{3}{ }^{-} \mathrm{E} 3$

Figura 3. Variación de la concentración de nitrato $\left(\mathrm{NO}_{3}^{-}\right)$a la salida de cada cámara (E1, E2 y E3), durante el tratamiento del efluente sintético (sacarosa + úrea), en los sistemas RBC aerobios de tres etapas, con respecto del tiempo de retención hidráulico aplicado (TRH)

La concentración de nitrato aumentó a medida en que el efluente pasaba entre las cámaras (Tabla 4 y Figura 3). Este comportamiento lo observaron Chen et al [6], durante el tratamiento de un RBC de tres etapas. Gupta y Gupta [14], en un reactor RBC de tres cámaras, observaron que para 
Ios cinco TRH estudiados (rango 18 a 6 h), a medida el efluente pasaba de una cámara a otra, la concentración de nitrato se incrementaba; también reportaron la presencia de nitrito, los cuales para TRH 18 h, se iban incrementando de una cámara a otra; comportamiento similar al obtenido en el presente estudio (Tabla 4). La proporción de nitrato siempre fue superior que la de nitrito (Tabla 4), cuya concentración mayor a la salida de los RBC se obtuvo cuando el sistema operó a la mayor carga orgánica estudiada (E3 = 261,27 $\mathrm{mgNO}_{3}$-/L).
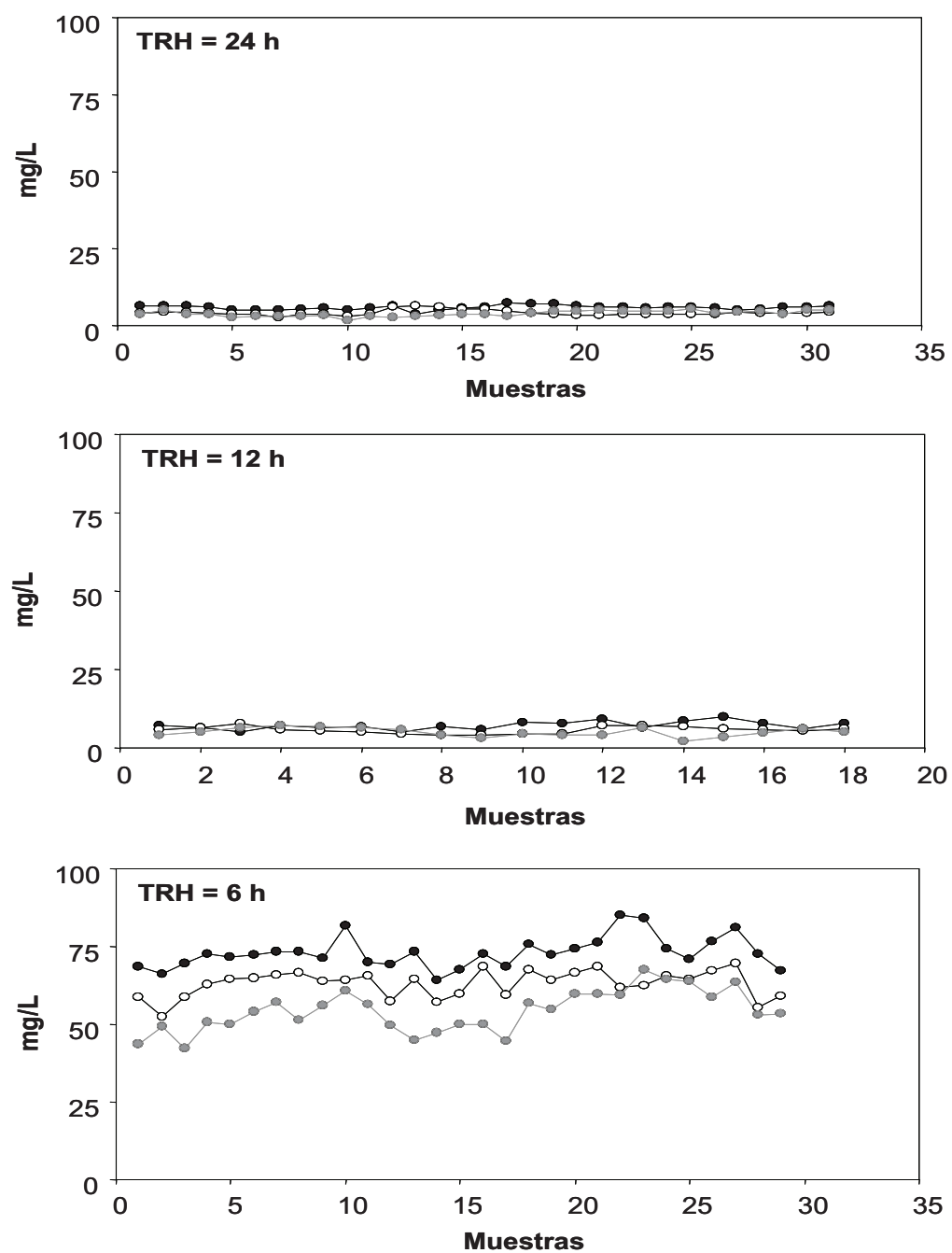

$$
\text { - } \mathrm{NH}_{4}^{+} \mathrm{E} 1-\mathrm{NH}_{4}^{+} \mathrm{E} 2-\mathrm{NH}_{4}^{+} \mathrm{E} 3
$$

Figura 4. Variación de la concentración de amonio $\left(\mathrm{NH}_{4}^{+}\right)$, a la salida de cada cámara (E1, E2 y E3), durante el tratamiento del efluente sintético (sacarosa + úrea) en los sistemas RBC aerobios de tres etapas, con respecto del tiempo de retención hidráulico aplicado (TRH) 
El nitrógeno amoniacal disminuyó en cada etapa, para todas las cargas orgánicas estudiadas. La concentración de $\mathrm{NH}_{4}{ }^{+}$en las cámaras, fue proporcional a la carga orgánica aplicada (Tabla 4 y Figura 4), es decir, se observó una mayor cantidad de amonio al TRH de 6 h. No hubo acumulación de nitrógeno amoniacal en el efluente de las cámaras para los diferentes TRH, lo cual evidencia la acción metabólica de las bacterias oxidadoras de amonio [6].
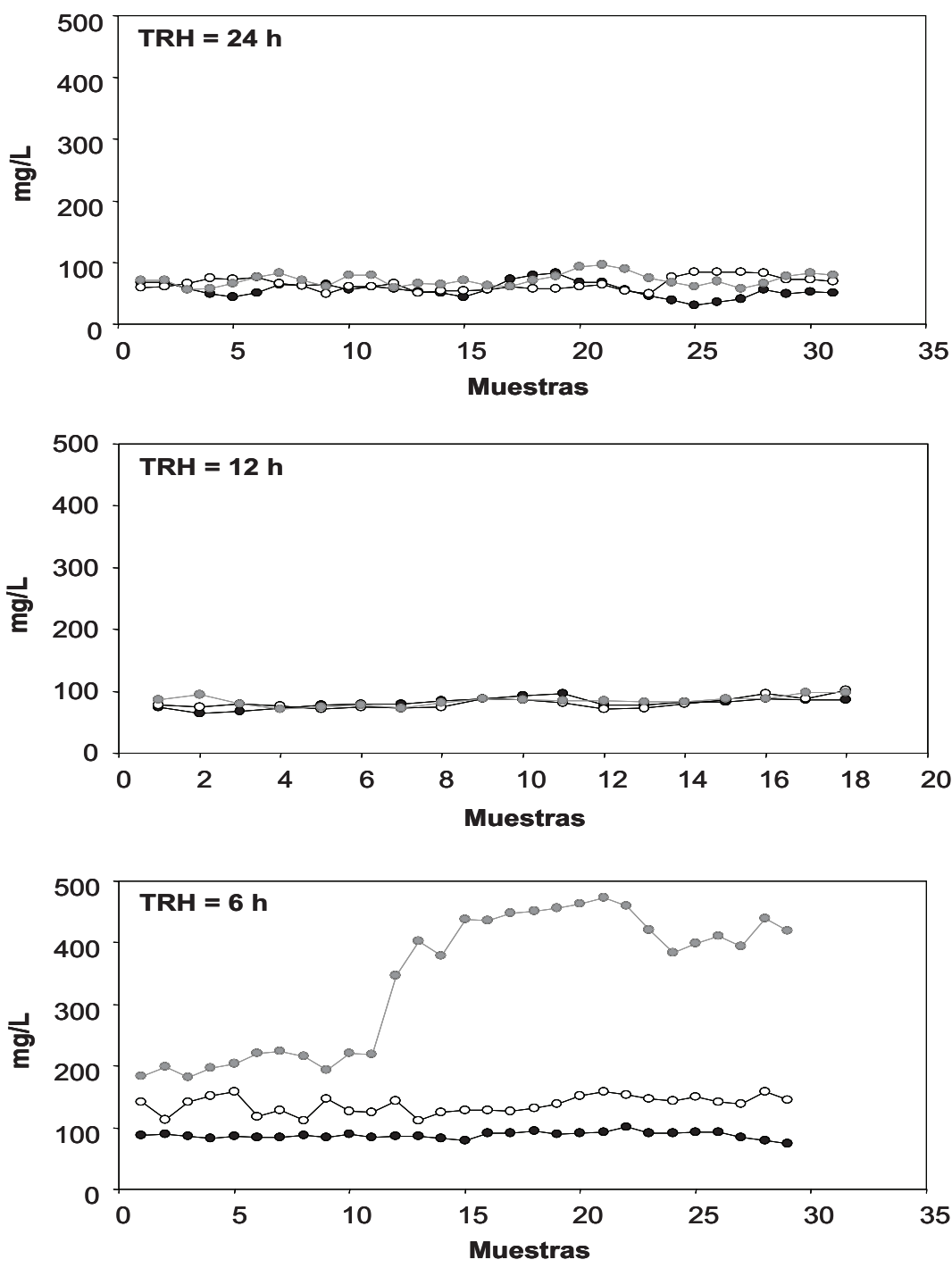

- N-total E1 $-\mathrm{N}$-total E2 $\rightarrow \mathrm{N}$-total E3

Figura 5. Variación de la concentración de nitrógeno total (N-total), a la salida de cada cámara (E1, E2 y E3), durante el tratamiento del efluente sintético (sacarosa + úrea), en los sistemas RBC aerobios de tres etapas, con respecto del tiempo de retención hidráulico aplicado (TRH) 
Para los TRH 24 y 12 h, la eficiencia de remoción de N-total fue respectivamente, de 66,92 y 62,95\%, considerando las tres cámaras. Sin embargo, cuando el TRH fue de 6 h, hubo mucha producción de nitrato, lo que originó un incremento de $\mathrm{N}$-total con respecto del influente de los sistemas. No obstante, en la primera cámara la eficiencia de remoción de N-total fue de 74,14; 64,39 y 60,83\% para los TRH 24, 12 y 6 h, respectivamente. Estas remociones son comparables con la reportada por Gupta y Gupta [15], quienes evaluaron el comportamiento de un RBC de tres cámaras, obteniendo una eficiencia de N-total en el primer compartimiento de 72,2\% para un TRH 24 h. Chen et al [6], por su parte, reportaron menores valores de eficiencia de remoción de $\mathrm{N}$-total que los obtenidos en el presente trabajo, para todos los TRH. Estos investigadores observaron que la eficiencia de remoción de N-total disminuía (61 a 40\%), a medida en que se reducía el TRH (9 a 5 h), y por ende, se incrementaba la CO, similar comportamiento al registrado en la presente investigación.

Las normas venezolanas de descarga en los cuerpos de agua superficiales, establecen como valores límites de $\mathrm{N}$-total $40 \mathrm{mg} / \mathrm{L}$ y de $\mathrm{NO}_{2}{ }^{-}+\mathrm{NO}_{3}{ }^{-} 10 \mathrm{mg} / \mathrm{L}$ [8]. En esta experimentación, para TRH 24 h, la concentración del N-total en E3 fue de 71,43 mg/L. Sin embargo, en la primera cámara fue de 55,83 mg/L, valor cercano al máximo establecido por la normativa. Tambien, se debe considerar que los sistemas experimentales no contaban con un sedimentador secundario, que pudiera contribuir a la disminución de estos valores.

\subsection{SÓLIDOS SUSPENDIDOS TOTALES}

La concentración de SST en el efluente final, aumentó conforme se incrementaba la CO del influente, y se observaron valores desde $68,6 \pm 8,2$ (E1, TRH 24 h), hasta 222,1 $\pm 23,3$ mg/L (E3, TRH 6 h), (Tabla 4). También fue notorio el aumento de los SST a través de las cámaras del reactor, como resultado del desprendimiento de la biopelícula de los discos. En general, la concentración de SST a la salida de los reactores excedió el valor máximo permisible de la legislación venezolana para la descarga en cuerpos de agua naturales $(80 \mathrm{mg} / \mathrm{L})$, [8]. Por tal motivo, se requiere implementar un postratamiento (sedimentador secundario), que garantice su disminución hasta los valores exigidos, según lo sugiere Griffin y Findlay [13].

\section{CONCLUSIONES}

La mayor remoción de DQO, se obtuvo para el TRH 12 h (CO global 11,68 gDOO/m².d), con una eficiencia general de $96,25 \%$. En la primera cámara de los reactores, se logró eliminar 95,89\% de la $\mathrm{DQO}_{\mathrm{T}}$ global aportada por el influente, y se produjo un efluente (E1), cuya concentración se encontraba muy por debajo del límite de descarga establecido por la legislación venezolana (350 mg/L).

Si bien durante el tratamiento no se logró reducir las concentraciones de $\mathrm{N}$-total a los límites de descarga establecidos (40 mg/L), en la primera cámara para TRH 24 h, la concentración del N-total 
fue de 55,83 mgN/L, valor cercano al exigido en la normativa. El incremento de los SST como resultado del desprendimiento de la biopelícula de los discos, sobrepasó el límite venezolano permisible para su descarga (80 mg/L), lo cual amerita la implementación de un postratamiento.

\section{AGRADECIMIENTOS}

El presente estudio fue cofinanciado por el Consejo de Desarrollo Científico, Humanístico y Tecnológico (CONDES), de La Universidad del Zulia (LUZ), Venezuela.

\section{REFERENCIAS BIBLIOGRÁFICAS}

[1] Alemzadeh I.; and Vossoughi F.; (2001). Biodegradation of toluene by an attached biofilm in a rotating biological contactor. En: Process Biochemistry, Vol. 36, pp. 707-711.

[2] Alemzadeh I.; Vossoughi F;; Houshmandi M.; (2002). Phenol biodegradation by rotating biological contactor. En: Biochemical Engineering Journal, Vol. 11, pp. 19-23.

[3] AMERICAN PUBLIC HEALTH ASSOCIATION (APHA), AMERICAN WATER WORKS ASSOCIATION (AWWA), WATER ENVIRONMENT FEDERATION (WEF), (1998). Standard methods for examination of water and wastewater (20 ed.). Washington: American Public Health Association 1015 Fifteenth Street, N.W.

[4] Banerjee Goutam, (1996). Phenol and thiocyanate-based wastewater treatment in RBC reactor. En: Journal of Environmental Engineering, Vol. 122, pp. 941-948.

[5] Castillo E., Vergara M., and Moreno Y., (2007). Landfill leachate treatment using a rotating biological contactor and an upward-flow anaerobic sludge bed reactor. En: Waste Management, Vol. 27, pp. 720-726.

[6] Chen Zhiqiang, Wen Oinxue, Wang Jianlong, LI Fang, (2006). Simultaneous removal of carbon and nitrogen from municipal-type synthetic wastewater using net-like rotating biological contactor (NRBC). En: Process Biochemistry, Vol. 41, pp. 2468-2472.

[7] Cortez S., Teixeira P., Oliveira R., and Mota M., (2008). Rotating biological contactors: a review on main factors affecting performance. En: Reviews Environmental in Science and Biotechnology, Vol. 7, pp. 155-172.

[8] DECRETO 883, (1995). Normas para la clasificación y el control de la calidad de los cuerpos de aguas y vertidos de efluentes líquidos. Gaceta Oficial de la República de Venezuela 5021 extraordinaria, sección III, pp.89-90. Caracas: 18 de diciembre. 
[9] Di Palma L., and Verdone N., (2009). The effect of disk rotational speed on oxygen transfer in rotating biological contactors. En: Bioresource Technology, Vol. 100, pp. 1467-1470.

[10] Dutta S., Hoffmann E., and Hahn H., (2007). Study of rotating biological contactor performance in wastewater treatment using multi-culture biofilm model. En: Water Science and Technology, Vol. 55, pp. 345-353.

[1 1] Eckenfelder William, (2000). Industrial water pollution control. 3 ed., New York: McGraw-Hill, $584 \mathrm{p}$.

[12] Fang H., and Yeong C., (1993). Biological wastewater treatment in reactors with fibrous packing. En: Journal of Environmental Engineering, Vol. 119, pp. 946-957.

[13] Griffin P., and Findlay G., (2000). Process and engineering improvements to rotating biological design. En: Water Science and Technology, Vol. 41, pp. 137-144.

[14] Gupta A.; and Gupta S., (1999). Simultaneous carbon and nitrogen removal in a mixed culture aerobic RBC biofilm. En: Water Research, Vol. 33, pp. 555-561.

[15] Gupta A., and Gupta S., (2001). Simultaneous carbon and nitrogen removal from high strength domestic wastewater in an aerobic RBC biofilm. En: Water Research, Vol. 35, pp. 1714-1722.

[16] Hanhan O., Orhon D., Krauth K., and Gunder B., (2005). Evaluation of denitrification potential of rotating biological contactors for treatment of municipal wastewater. En: Water Science and Technology, Vol. 51, pp. 131-139.

[17] Herman Susana, (1997). Proceso de salinización en el Lago de Maracaibo. Instituto para el Control y la Conservación del Lago de Maracaibo (ICLAM), 109 p.

[18] Hiras Demetrios, Manariotis Ioannis, and Grigoropoulus Sotirios, (2004). Organic and nitrogen removal in a two-stage rotating biological contactor treating municipal wastewater. En: Bioresource Technology, Vol. 93, pp. 91-108.

[19] Israni Sameer, Koli Shrikant, Patwardhan A., Melo J., and D'souza S., (2002). Phenol degradation in rotating biological contactors. En: Journal of Chemical Technology and Biotechnology, Vol. 77, pp. 1050-1057.

[20] Martín-Cereceda Mercedes, Serrano Susana, and Guinea Almudena, (2000). Biofilm communities and operational monitoring of a rotating biological contactor system. En: Water Science and Technology, Vol. 43, pp. 247-253. 
[21] Metcalf Y. Eddy, (2003). Wastewater engineering: treatment, disposal and reuse. 4 ed., New York: McGraw-Hill, 1771 p.

[22] Najafpour Gashem, Naidu Punita, and Kamaruddin Azlina, (2002). Rotating biological contactor for biological treatment of poultry processing plant wastewater using Saccharomyces cerevisiae. En: ASEAN Journal of Chemical Engineering, Vol. 2, pp. 1-6.

[23] Najafpour Ghasem, Yieng Hii, Younesi Habibollah, and Zinatizadeh Aliakbar, (2005). Effect of organic loading on performance of rotating biological contactors using palm oil mill effluents. En: Process Biochemistry, Vol. 40, pp. 2879-2884.

[24] Najafpour Ghasem, Zinatizadeh A., and Lee L., (2006). Performance of a three-stage aerobic RBC reactor in food canning wastewater treatment. En: Biochemical Engineering Journal, Vol. 30, pp. 297-302.

[25] Nowak O., (2000). Upgrading of wastewater treatment plants equipped with rotating biological contactors to nitrification and P removal. En: Water Science and Technology, Vol. 41, pp. 145-153.

[26] Osho A., Mabekoje O., and Bello O., (2010). Preliminary evaluation of wastewater effluents from two food companies in Nigeria. En: African Journal of Microbiology Research, Vol. 4, pp. 1395-1399.

[27] Patwardhan A., (2003). Rotating biological contactors: a review. En: Industrial \& Engineering Chemistry Research, Vol. 42, pp. 2035-2051.

[28] Satinder K., and Gupta S., (2000). Biodegradation of trichloroethylene in a rotating biological contactor. En: Water Research, Vol. 34, pp. 4207-4214.

[29] Schmidt I., Sliekers O., Schmid M., Bock E., Fuerst J., Kuenen J., Jetten M., and Strous M., (2003). New concepts of microbial treatment processes for the nitrogen removal in wastewater. En: FEMS Microbiology Reviews, Vol. 27, pp. 481-492.

[30] Shrivastava Pooja, and Soni Anupam, (2012). Treatment of rice mill effluent for pollution control by electrocoagulation. En: Journal of Chemical, Biological and Physical Sciences, Vol. 2, pp. 480-483.

[31] Spengel Douglas, and Dzombak David; (1991). Treatment of landfill leachate with rotating biological contactors: bench-scale experiments. En: Research Journal WPCF, Vol. 63, pp. 971-980. 
[32] Tchobanoglous G., and Burton F., (1991). Wastewater engineering: treatment, disposal and reuse. 3 ed., Metcalf \& Eddy. New York: McGraw-Hill.

[33] Teixeira Pilar, and Oliveira Rosário; (2001). Denitrification in a closed rotating biological contactor: effect of disk submergence. En: Process Biochemistry, Vol. 37, pp. 345-349.

[34] Torkian Ayoob, Yazdani O., and Alinejad K., (2003). Treatability evaluation of municipal wastewater and anaerobically-treated industrial effluent in a rotating biological contactor. En: IJE Transactions B: Applications, Vol. 16, pp. 143-154.

[35] Torkian Ayoob, Alinejad K., and Hashemian S., (2003). Posttreatment of upflow anaerobic sludge blanket-treated industrial wastewater by a rotating biological contactor. En: Water Environment Research, Vol. 75, pp. 232-237. 